\title{
Revealing the contest between triplet-triplet exchange and triplet-triplet energy transfer coupling in correlated triplet pair states in singlet fission
}

\author{
Vibin Abrahan* and Nicholas J. Mayhal ${ }^{\dagger}$ \\ Department of Chemistry, Virginia Tech, Blacksburg, VA 24060, USA
}

\begin{abstract}
Understanding the separation of the correlated triplet pair state ${ }^{1}(\mathrm{TT})$ intermediate is critical for leveraging singlet fission to improve solar cell efficiency. This separation mechanism is dominated by two key interactions: (i) the exchange interaction $(K)$ between the triplets which leads to the spin splitting of the biexciton state into ${ }^{1}(\mathrm{TT}),{ }^{3}(\mathrm{TT})$ and ${ }^{5}(\mathrm{TT})$ states, and (ii) the triplet-triplet energy transfer integral $(t)$ which enables the formation of the spatially separated (but still spin entangled) state ${ }^{1}(\mathrm{~T} \ldots \mathrm{T})$. We develop a simple ab initio technique to compute both the biexciton exchange $(K)$ and biexciton transfer coupling. Our key findings reveal new conditions for successful correlated triplet pair state dissociation. The biexciton exchange interaction needs to be ferromagnetic or negligible to the triplet energy transfer for favourable dissociation. We also explore the effect of chromophore packing to reveal geometries where these conditions are achieved for tetracene.
\end{abstract}

\section{TOC Graphic:}

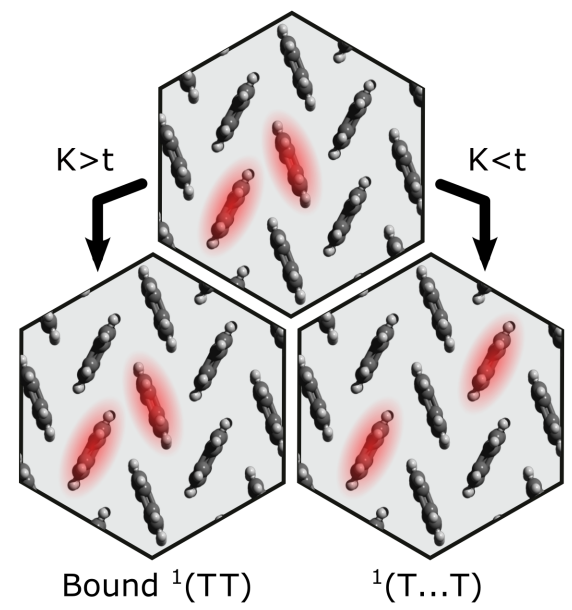

\section{INTRODUCTION}

Singlet fission 14 is a spin allowed process where an excited singlet state $\left(S_{1}\right)$ is converted to two low energy triplet states $\left(T_{1}+T_{1}\right)$. Although discovered around 50 years ago, it was recently shown that the process can be used to overcome the Shockley-Queisser limit for efficiency of single junction organic solar cells. ${ }^{5}$ The singlet fission mechanism involves a singlet excited state $\left(S_{1}\right)$ forming a spin coupled triplet excited state ${ }^{1}(\mathrm{TT})$ before splitting into two independent triplet states.113

The correlated triplet pair state ${ }^{1}(\mathrm{TT})^{6}{ }^{6}$ serves as the key spin conserving intermediate between the final triplet states and the initial singlet state for the singlet fission

*Electronic address: vibin1@vt.edu

${ }^{\dagger}$ Electronic address: nmayhall@vt.edu process ${ }^{110}$ While the separation of the ${ }^{1}$ (TT) to individual triplets was initially thought to be a fast process in $f s$ region, recent studies have shown it to stay correlated until ns timescales $11-15$ Being a long lived state, multiple competing decay pathways exist for the ${ }^{1}(\mathrm{TT})$, beyond simply separating into individual triplets. The formation of the ${ }^{5}$ (TT) state, which is the quintet coupled triplet pair state is also reported recently $12 \mid 16-20$ One of the main advantages of forming the ${ }^{5}(\mathrm{TT})$ state is that it doesn't have any decay channel other than to separate into individual triplets unlike ${ }^{1}(\mathrm{TT})$ or ${ }^{3}(\mathrm{TT}) \stackrel{21}{2}$ These long lived ${ }^{1}$ (TT) states ${ }^{13}$ might also have important applications in quantum information. ${ }^{22}$ Being a multichromophoric process, the interaction between molecules is very important for the singlet fission process $\frac{18|23| 24}{\text { To }}$ realize the ideal of fast singlet-fission, the electronic interaction between chromophores should be strong enough for the triplet states to be entangled, yet not too strong such that the ${ }^{1}(\mathrm{TT})$ becomes a trap state.

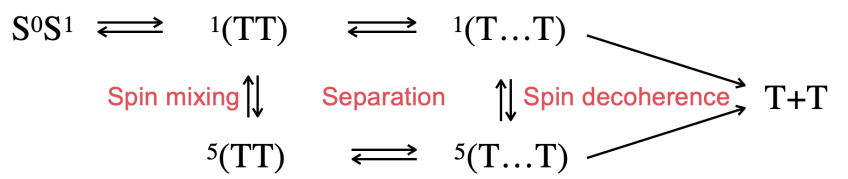

FIG. 1: Schematic representation of the mechanism of singlet fission dissociation process.

The triplet-triplet exchange interaction $(K)$ provides insight into the energy manifold of the different spin states of biexcitonic (TT) character. This interaction is usually antiferromagnetic, ${ }^{25126}$ leading to the following state energy ordering: ${ }^{1}(\mathrm{TT})<{ }^{3}(\mathrm{TT})<{ }^{5}(\mathrm{TT})$. Closely packed (or covalently bonded) chromophores often exhibit large exchange interaction ${ }^{12}$ Usually, this close packing increases the electronic coupling between the initial singlet state and the ${ }^{1}(\mathrm{TT})$ and can lead to decoherence via triplet-triplet annihilation depending on 
the energy level of the initial bright state and the ${ }^{1}$ (TT) state ${ }^{1127}$ For energy levels that favor TTA, it has been shown that lowering this coupling can lead to higher yields of the separated triplets from the 1TT in case of intramolecular singlet fission. $17726 / 28 / 29$

Even after establishing the spin-state orderings of (TT), the dissociation of the correlated triplet pair state is still not well understood. Spatially separated yet spin entangled ${ }^{1}(\mathrm{~T} \ldots \mathrm{T})$ states have recently been proposed as a possible intermediate ${ }^{30}$ and have subsequently been characterized experimentally ${ }^{3132}$ The triplet-triplet energy transfer (or Dexter) ${ }^{33}$ mechanism has been proposed for the formation of these (T...T) states 31132134135 The triplet-triplet energy transfer rate largely depends on the square of the triplet-triplet energy transfer coupling $(t), \frac{36 \sqrt[38]{38}}{3}$ wich is an exchange type interaction and decays exponentially with distance between donor and acceptor (similar to $K){ }^{39 / 40}$ Formation of the ${ }^{1}(\mathrm{~T} \ldots \mathrm{T})$ has recently been shown to improve the final triplet yield in covalently linked tetracene trimers and tetramers $\underline{41}$

The purpose of this manuscript is two fold: First, we present an efficient ab initio-based technique to characterize the multi-excitonic states in terms of a simple model Hamiltonian consisting of physically meaningful quantities, $t$ and $K$. This model Hamiltonian, in turn, allows us to identify values of $t$ and $K$ which lead to favorable energetics for dissociation of the (TT) state into independent triplet excitons. Second, we perform numerical calculations to resolve the connection between chromophore packing and the $t$ and $K$ parameters to identify which geometries might be preferable for efficient triplet separation.

Theoretical calculations $\$ 22 / 274251$ play an important role in characterizing the ${ }^{1}(\mathrm{TT})$ state, with its first theoretical report by Zimmerman and coworkers 10 much before its first experimental detection ${ }^{52}$ Being a doubly excited state, conventional single excitation methods (e.g., CIS, TDDFT, and RPA) cannot represent this state ${ }^{53}$ Hence, calculations require very expensive multireference methods like CASSCF ${ }^{54}$ or MRPT ${ }^{555}$. Spin flip ${ }^{56}$ methods are an alternate approach where the multiconfigurational systems can be studied by starting from a well-defined high spin single reference. In this work, we use a single spin flip method to obtain the two key electronic couplings ( $K$ and $t$ ) needed to describe the multiexciton space. We present numerical evidence that the multiexciton space is well represented using these two couplings.

\section{METHODS}

Complete active space spin-flip (CAS-SF) and restricted active space spin-flip (RAS-SF) methods $57+59$ have been successfully used to study the singlet fission process ${ }^{3460} \frac{64}{6 v e n}$ though the RAS-SF calculation can produce reliable results for medium sized systems, the calculations get intractable as the number of chro- mophores increases. The reason, is that the number of spin-flip excitations increases combinatorially with number of chromophores.

Recently, our group proposed a hybrid numerical/analytical method where only a simple 1-spin flip calculation is needed to obtain the biexciton manifold instead of factorially scaling $n$ spin flips ${ }^{25}$ This is carried out by extracting a spin Hamiltonian using the single spin flip method and subsequently using the spin Hamiltonian to form the excited states, hence having the singly, doubly, and higher excited triplet states ${ }^{[65}$ However, having extracted a spin Hamiltonian does not fully alleviate the scaling problem. Even though the spin Hamiltonian is smaller compared to the ab initio Hamiltonian, the scaling is still factorial with system size. In this work, we further simplify this Hamiltonian to form a biexciton model, which only involves the doubly excited space of the spin Hamiltonian. We further demonstrate that this Hamiltonian can be formed solely by using the biexciton exchange and the biexciton hopping term.

\section{A. Setup}

While spin models have commonly been used to represent the coupled (TT) state ${ }^{117124166}$ these models mapped each chromophore to a spin 1 site. While these approaches were able to describe the dimer systems, the 1to-1 mapping prevented the descriptions of biexcitons on model systems containing more than two chromophores. In our recent work $\frac{\sqrt{25}}{,}$ a 1-to-2 mapping was introduced, wherein a chromophore system with $n$ chromophores can be cast as a $2 n$ site spin lattice, allowing application to systems larger than chromophore dimers. This approach, represents each chromophore as having two $S=\frac{1}{2}$ spins, rather than one $S=1$ spin. In brief, this process involves computing the high spin ROHF (or DFT) calculation with multiplicity $2 n+1$, and then carrying out a single spin flip calculation (CAS-SF, RAS-SF, SF-TDDFT) to obtain the lowest $2 n$ eigenvectors in the $2 n-1$ multiplicity space (Figure $2 \mathrm{a}$ ). The singly occupied orbitals are localized (Figure 25) which organizes the determinant basis into either neutral or ionic configurations. We finally project the 1-SF eigenstates into the neutral determinant basis and reorthogonalize to obtain a new basis. The ab initio eigenvalues can be represented as a Bloch effective Hamiltonian matrix ${ }^{65|67| 68}$ and the spin half exchange interaction can be obtained from the off diagonal elements of this effective Hamiltonian.25

For a dimer system as shown in Figure $2 a$, starting from a quintet guess and using a single spin flip gives the two triplet states, ${ }^{3}(\mathrm{TT})$ and ${ }^{5}(\mathrm{TT})$ in the $m_{s}=+1$ space. Using these four states, we can form the $\operatorname{spin}=\frac{1}{2}$ Hamiltonian using the procedure as mentioned in Ref. [25].

$$
\hat{H}^{\text {spin }}=-2 \sum_{\mathrm{ij}} J_{\mathrm{ij}} \hat{S}_{\mathrm{i}} \hat{S}_{\mathrm{j}}
$$


a)

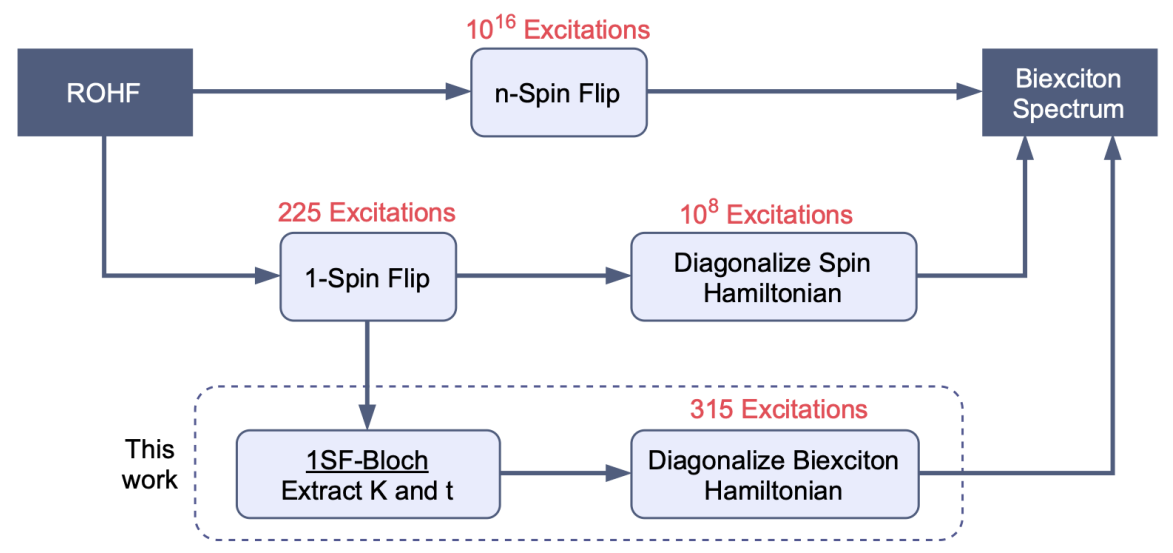

b)

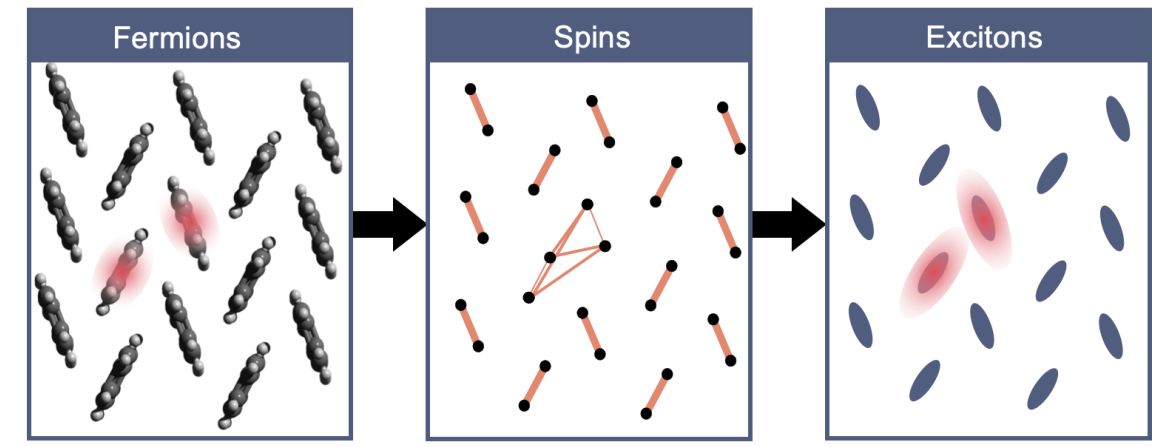

c)
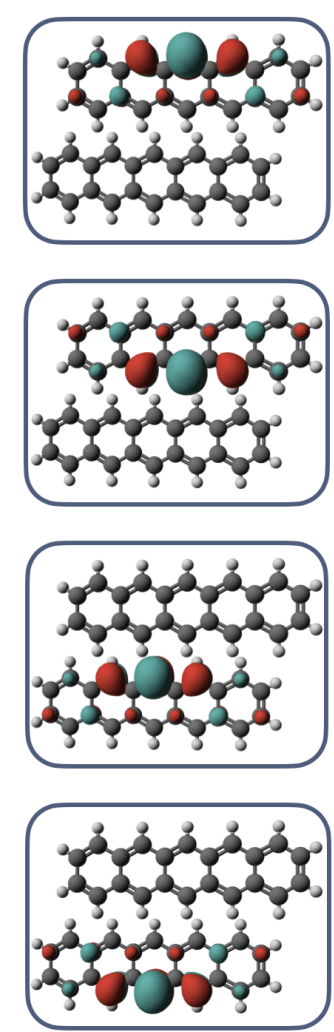

FIG. 2: a) The final energy levels/biexciton spectrum for the bound triplet state energy manifold can be obtained using CASnSF and also by using CAS-1SF+ spin Hamiltonian. In this work we introduce the 1SF-Bloch method to obtain the biexciton spectrum which scales polinomially instead of exponentially. The numbers in red corresponds to the number of variables in the wavefunction for a system with 15 chromophores. b) Illustration showing the transformation from the molecular lattice into spin lattice and the subsequent biexciton model. c) Singly occupied localized orbitals in the singly occupied active space for the pentacene dimer.

The ability to construct an accurate spin model offers multiple advantages such as deeper conceptual insight, numerical efficiency, and ease of implementation of periodic boundary conditions. In the spin Hamiltonian, the interactions between sites which are on the same chromophore are much largeer than the interchromophore interactions. Hence we can write the Hamiltonian as

$$
\hat{H}=\sum_{I} \hat{H}_{I}+\sum_{I J} \hat{H}_{I J}
$$

where $I$ and $J$ are chromophore indices. The intra chromophore exchange interactions are much larger than inter terms, and hence we can define the zeroth order as the intra terms. These intra chromophore interactions give an estimate of the excitation energy for a completely localized triplet state on each chromophore. We can diagonalize $\sum_{I} \hat{H}_{I}$ to form exciton subspaces corresponding to single, double and higher excited triplet states. The energy manifold of each of these excited subspaces is well separated from the manifolds of other subspaces. 25

Since we are interested in the biexciton space, we can therefore ignore the effect of other states including the reference lowest energy state and other excited manifolds. We can diagonalize the Hamiltonian in the biexciton space to obtain the energies of the (TT) states. We test this model by analyzing the biexciton energy by diagonalizing the spin Hamiltonian and using our model for a tetracene heptamer in Section IIIA. By removing the ground state from the diagonalization, this approximation provides size consistent energies ${ }^{69}$ and can be extended to larger systems. This diabatic biexciton space has mainly two types of interactions, the exchange interaction between the triplet states and the energy transfer coupling. We will refer to our method to obtain the exchange interaction and the transfer coupling as 1SFBloch approach.

\section{B. Biexciton Exchange Integral}

The decoherence of the ${ }^{1}(\mathrm{TT})$ state for molecular dimers depends largely on the inter triplet exchange interaction $(K){ }^{25 / 70 / 71}$ For a chromophore dimer system, 

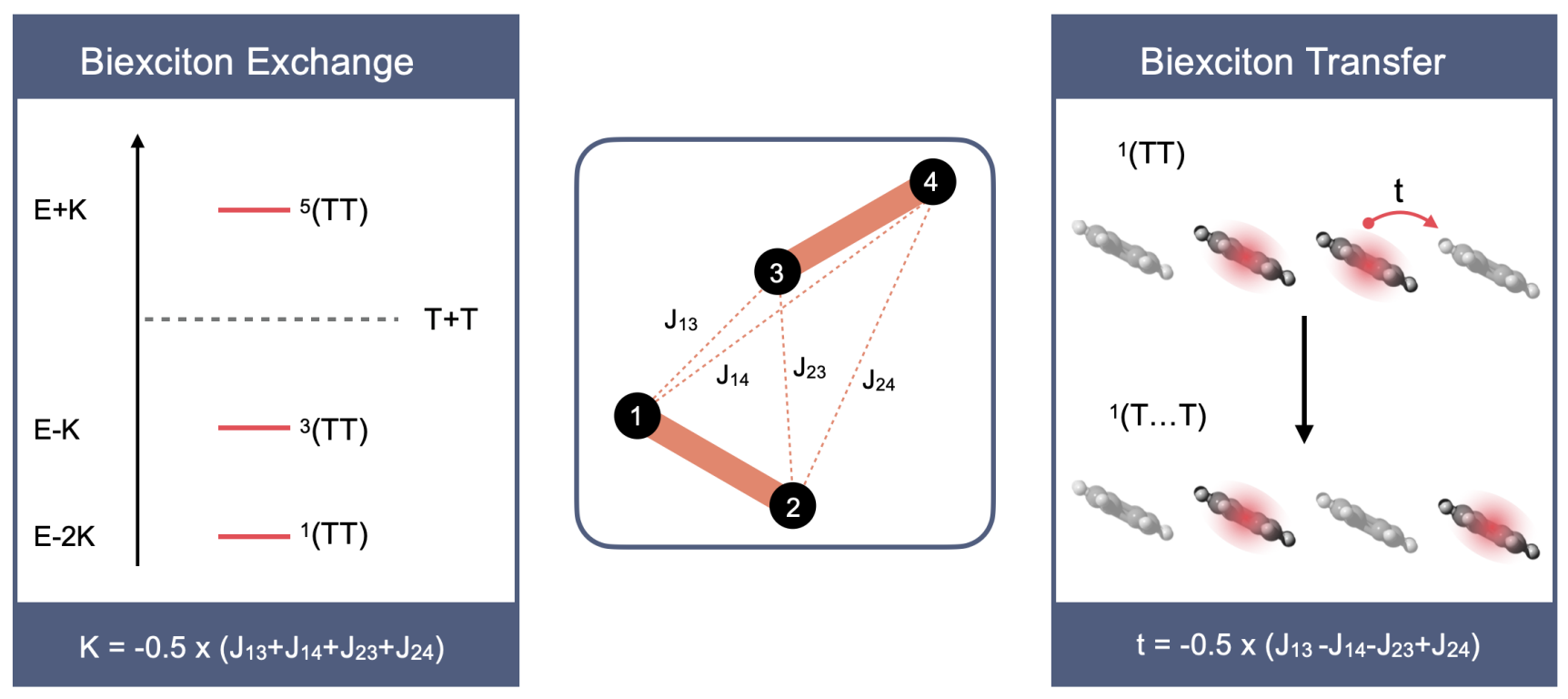

FIG. 3: The 4 site spin model for a two chromophore system. The biexciton exchange integral splits the three spin components energetically. The biexciton transfer integral plays an important role in formation of ${ }^{1}(\mathrm{~T} . . \mathrm{T})$ from ${ }^{1}(\mathrm{TT})$.

we can compute the $K$ value from the spin Hamiltonian derived using the 1SF calculation. The biexciton basis for a dimer system in the $m_{s}=0$ space can be formed using the zero order space $\left|\mathrm{T}^{+} \mathrm{T}^{-}\right\rangle,\left|\mathrm{T}^{0} \mathrm{~T}^{0}\right\rangle$ and $\left|\mathrm{T}^{-} \mathrm{T}^{+}\right\rangle$ where the first index corresponds to chromophore $\mathrm{A}$ and the second index corresponds to chromophore B.

The $\left|\mathrm{T}^{-}\right\rangle,\left|\mathrm{T}^{0}\right\rangle$ and $\left|\mathrm{T}^{+}\right\rangle$can be written as

$$
\begin{aligned}
& \left|\mathrm{T}^{-}\right\rangle=|\beta \beta\rangle \\
& \left|\mathrm{T}^{0}\right\rangle=\frac{1}{\sqrt{2}}|\alpha \beta+\beta \alpha\rangle \\
& \left|\mathrm{T}^{+}\right\rangle=|\alpha \alpha\rangle
\end{aligned}
$$

The matrix element between any two of these states can be evaluated quite easily for the spin lattice. For example, the matrix element between the $\left|\mathrm{T}^{+} \mathrm{T}^{-}\right\rangle$and $\left|\mathrm{T}^{0} \mathrm{~T}^{0}\right\rangle$ in terms of the spin half exchange matrix element comes as,

$$
\left\langle\mathrm{T}^{+} \mathrm{T}^{-}|H| \mathrm{T}^{0} \mathrm{~T}^{0}\right\rangle=-\frac{1}{2}\left(J_{13}+J_{14}+J_{23}+J_{24}\right)
$$

Here site 1 and 2 are in chromophore A and site 3 and 4 are in chromophore B (Figure 3 .

This integral is the triple-triplet exchange interaction $(K)$ between the two triplet states in the biexciton space. Hence we can write $K$ in terms of the inter chromophore exchange interactions.

$$
K_{\mathrm{AB}}=-\frac{1}{2}\left(J_{13}+J_{14}+J_{23}+J_{24}\right)
$$

The $3 \times 3$ Hamiltonian for the dimer using basis $\left|\mathrm{T}^{+} \mathrm{T}^{-}\right\rangle,\left|\mathrm{T}^{0} \mathrm{~T}^{0}\right\rangle$ and $\left|\mathrm{T}^{-} \mathrm{T}^{+}\right\rangle$can be written as ,

$$
\hat{H}=\left[\begin{array}{ccc}
E-K_{\mathrm{AB}} & K_{\mathrm{AB}} & 0 \\
K_{\mathrm{AB}} & E & K_{\mathrm{AB}} \\
0 & K_{\mathrm{AB}} & E-K_{\mathrm{AB}}
\end{array}\right]
$$

where $\mathrm{E}$ is the reference energy for the biexciton space, and $K_{\mathrm{AB}}$ is the exchange interaction between the two triplets in chromophores A and B. Diagonalization of this Hamiltonian leads to the three spin components of the multiexciton, ${ }^{1}(\mathrm{TT}),{ }^{3}(\mathrm{TT})$ and ${ }^{5}(\mathrm{TT})$ with energies $E-2 K_{\mathrm{AB}}, E-K_{\mathrm{AB}}$ and $E+K_{\mathrm{AB}}$ respectively and eigenvectors:

$$
\begin{gathered}
|1(\mathrm{TT})\rangle=\frac{1}{\sqrt{3}}\left(\left|\mathrm{~T}^{+} \mathrm{T}^{-}\right\rangle-\left|\mathrm{T}^{0} \mathrm{~T}^{0}\right\rangle+\left|\mathrm{T}^{-} \mathrm{T}^{+}\right\rangle\right) \\
\left.\left.\right|^{3}(\mathrm{TT})\right\rangle=\frac{1}{\sqrt{2}}\left(\left|\mathrm{~T}^{+} \mathrm{T}^{-}\right\rangle-\left|\mathrm{T}^{-} \mathrm{T}^{+}\right\rangle\right)
\end{gathered}
$$

$$
|5(\mathrm{TT})\rangle=\frac{1}{\sqrt{6}}\left(\left|\mathrm{~T}^{+} \mathrm{T}^{-}\right\rangle+2\left|\mathrm{~T}^{0} \mathrm{~T}^{0}\right\rangle+\left|\mathrm{T}^{-} \mathrm{T}^{+}\right\rangle\right)
$$

The $K$ parameter also plays an important role that it contributes to the exciton binding energy $\left(E_{b}\right)$ defined as the energy difference between ${ }^{1}(\mathrm{TT})$ and separated triplets. There are studies reporting the biexciton binding energy as low as neV ${ }^{72 / 73}$ to as high as 100's 
of meV!7475 Connecting singlet fission chromophores through a covalent linker ${ }^{176}$ increases the interaction between the chromophores and usually results in large $E_{b}$ compared to crystals. ${ }^{26}$ One of the major limitations with covalently connected chromophores is that the rate of recombination to form the singlet state increases, diminishing the final triplet population $1127 / 77$ Recently, Basel et. al studied singlet fission using a non-conjugated linker between the pentacene monomers thereby decreasing the inter-chromophore exchange interaction. ${ }^{16]}$ Another approach is by connecting the singlet fission chromophores through meta posotion of a bridging benzene molecule to change the sign of the exchange interaction! ${ }^{1726}$ The final triplet yield is increased in such cases. Hence it can be said that low/ ferromagnetic $K$ value can lead to improved triplet yield.

The exchange interaction (Equation. 5) derived here is similar to the two orbital representations using the diabatic pseudo canonical orbitals with HOMO and LUMO orbitals in both the chromophore. The exchange interaction comes down to the sum of exchange integral between the orbitals in that representation as well. $6[30 \mid 78$

\section{Biexciton Transfer Integral}

Recent studies have proposed that the decoherence of the ${ }^{1}$ (TT) state occurs by evolving into the ${ }^{1}(\mathrm{~T} \ldots \mathrm{T})$ state

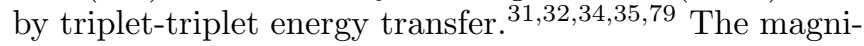
tude of TT coupling, which is exchange type in nature depends exponentially on the distance between triplet donor and acceptor $\frac{33|80| 81}{3}$ Theoretical methods using singly excited methods have been previously used to estimate the triplet-triplet energy transfer integral using the fragment spin difference method ${ }^{37 / 40}$ Charge transfer states are also important for the triplet-triplet energy

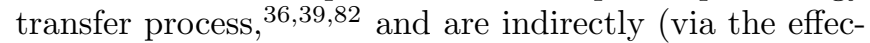
tive interactions) included in our model ${ }^{25}$

Given the spin Hamiltonian, the triplet-triplet hopping can be extracted easily by looking at the biexciton or single exciton space. For example, consider a three chromophore system, where the biexciton can shift from one pair to another. For the trimer, the size of the biexciton space is ${ }^{3} C_{2} \times 3=9$ which includes three of each ${ }^{1}(\mathrm{TT})$, ${ }^{3}(\mathrm{TT})$ and ${ }^{5}(\mathrm{TT})$. By closely analyzing the Hamiltonian, it can be seen that the biexciton subspace contains both the exchange interaction $(K)$ between the chromophores and a new integral corresponding to triplet transfer, $t$. This becomes evident when we work with the spin coupled biexciton space. This makes the Hamiltonian block diagonal with dense singlet, triplet and quintet blocks. The resulting Hamiltonian after spin coupling has the $K$ terms in the diagonal.

The triplet-triplet hopping between two diabatic biexcitons ${ }^{1}(\mathrm{TT})_{A C}$ and ${ }^{1}(\mathrm{TT})_{B C}$ can be defined as the matrix element between the two states using the spin Hamil- tonian.

$$
t_{\mathrm{AB}}=\left\langle{ }^{1}(\mathrm{TT})_{B C}|\hat{H}|^{1}(\mathrm{TT})_{A C}\right\rangle
$$

Similar to $K$, the $t$ parameter between chromophore A and $\mathrm{B}$ can be written as a linear combination of the inter chromophore exchange terms.

$$
t_{\mathrm{AB}}=\frac{1}{2}\left(-J_{13}+J_{14}+J_{23}-J_{24}\right)
$$

For the trimer, the "singlet-biexciton" block of the Hamiltonian can be represented as using basis of ${ }^{1}(\mathrm{TT})_{A B},{ }^{1}(\mathrm{TT})_{A C}$ and ${ }^{1}(\mathrm{TT})_{B C}$ can be written as,

$$
\hat{H}\left[{ }^{1}(\mathrm{TT})\right]=\left[\begin{array}{ccc}
E-2 \mathrm{~K}_{A B} & \mathrm{t}_{B C} & \mathrm{t}_{A C} \\
\mathrm{t}_{B C} & E-2 \mathrm{~K}_{A C} & \mathrm{t}_{A B} \\
\mathrm{t}_{A C} & \mathrm{t}_{A B} & E-2 \mathrm{~K}_{B C}
\end{array}\right]
$$

The transfer integral for the quintet and triplet multiexcitons are the same as the singlet state. The off diagonal elements for the model Hamiltonians for those states look exactly same as Equation 12. We compare the triplettriplet transfer coupling obtain using the 1SF-Bloch with the traditional CIS based approach $\frac{3783}{10}$ in supporting information. The energy cleft method has also been used recently to help triplet-triplet separation in case of intramolecular singlet fission. 84

\section{Biexciton Hamiltonian}

By using the 1SF-Bloch method, we have derived parameters that model the biexciton subspace. The (TT) energy manifold for all three spin states can be obtained using the biexciton space. The final biexciton Hamiltonian for the ${ }^{1}(\mathrm{TT})$ state using the two parameters can be written as:

$$
\begin{aligned}
\hat{\mathbf{H}}_{(\mathrm{TT})}= & \sum_{A, B}-2 K_{\mathrm{AB}}|A, B\rangle\langle A, B| \\
& +\sum_{A B C} t_{A B}|A, C\rangle\langle C, B| .
\end{aligned}
$$

The basis $|A, B\rangle$ is the biexciton state where chromophores A and B are in their triplet excited states. The full biexciton Hamiltionian with all three spin states for an $n$ chromophore system scales as $3 \times{ }^{n} C_{2}$. Hence, the dimension of this Hilbert space scales quadratically with the number of chromophores compared to the factorial scaling for the parent spin Hamiltonian. In this work we diagonalize this Hamiltonian to look at the biexciton energy spectrum and understand the effect of $\mathrm{K}$ and $\mathrm{t}$ on the biexciton spectrum. Numerically these couplings are small and usually in meV scale which is much smaller than the excitation energies. 
With the aide of the 1SF-Bloch model, we can now use a simple 1-spin flip calculation from a high spin reference to simultaneously obtain both the biexciton exchange and transfer integrals. In terms of the impact on singlet-fission triplet yield, the two parameters play opposing roles. Large antiferromagnetic exchange interaction (positive $K$ ) can lead to ${ }^{1}$ (TT) being a strongly bound state and reduces final triplet yield. Meanwhile having a larger hopping integral compared to the exchange should facilitate the formation of spatially separate (T...T) state. Hence, we expect systems with large $t$ and small/negative $K$ to facilitate in the dissociation of the biexciton state.

Even though both of these interactions are on the same scale, their relative magnitudes can change based on the interaction between the chromophores. Since, the biexciton exchange interaction is a sum of the spin Hamiltonian terms, it does not really become zero for molecular assemblies when all sites have antiferromagnetic interaction. The biexciton transfer has both positive and negative contributions from the spin half exchange interactions meaning that there is an orientational dependence. For example, having two chromophores perpendicular to each other cancels the exciton transfer integral while the biexciton exchange interaction stays non zero.

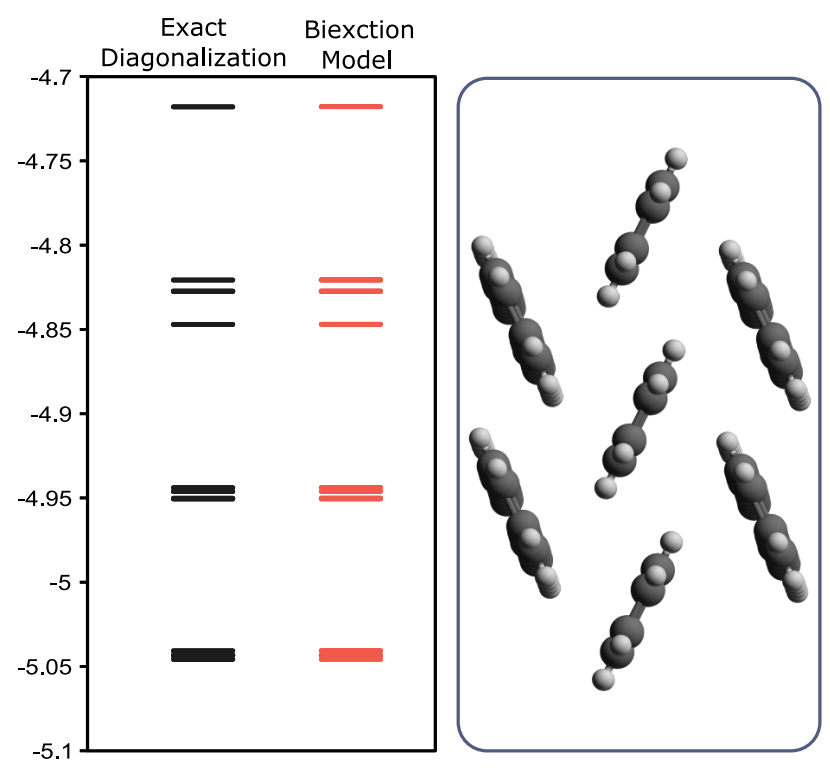

FIG. 4: Comparison of the biexciton model with the exact result from the spin Hamiltonian for a system with seven tetracene units.

\section{RESULTS AND DISCUSSION}

We focus on three different aspects of the biexciton model proposed in this work. In Section IIIA, we compare numerical data for the biexciton model to the data from the actual diagonalization of the spin Hamiltonian for a large chromophore assembly. Secondly, in Section IIIB we analyze the effect of different ratios of exchange to transfer parameter and look at the biexciton spectrum for a model 1D system. Finally, in Section IIIC we investigate how the exchange and transfer parameters vary as we change the chromophore orientations and suggest geometric orientations where improved triplet dissociation is possible using our model.

\section{A. Tetracene 7-mer}

As described above, the mapping of the ab initio Hamiltonian to a quantum spin lattice does not completely remove the combinatorial complexity of the problem. While the dimension of the spin-Hamiltonian Hilbert space is indeed smaller (it is the square root of the fermionic problem), it is still intractable to diagonalize for large systems. However, because we are only concerned with the biexciton states (in contrast to tri-excitons and so forth), we propose to build and diagonalize the spin-Hamiltonian only the the biexcitonic manifold. This not only reduces the computational cost to polynomial scaling, but also restores size consistency by removing the ground state from the diagonalization space. 
In this section, we evaluate the magnitude of the errors obtained by diagonalizing in only the biexcitonic subspace instead of the full space. In our previous work, 25 we computed the biexciton spectrum using exact diagonalization (ED) of the full spin Hamiltonian for a large cluster with seven tetracene monomers. In Figure 4 we show the comparison of the ${ }^{1}$ (TT) spectrum using ED and the biexciton model Hamiltonian proposed in this paper. There are a total of $21^{1}(\mathrm{TT})$ states for this system. As seen in Figure 4, the spectrum obtained from diagonalizing only the biexcitonic manifold is essentially indistinguishable from the exact diagonalization. We have also derived a rigorous downfolding using quasi-degenerate perturbation theory, but since the zeroth-order results presented here are accurate enough, we only provide these slighly more accurate results in the supplementary information.

The broadening of the biexciton spectrum is largely due to static disorder of the tetracene monomers (which would vanish if periodic boundary conditions were used in the ab initio calculations, which will be a focus of future work) and other acenes having two different geometries in the herringbone crystal structure. ${ }^{85}$ The energy gap between these two different structures is as large as 50 $\mathrm{meV}$ which is larger than the exchange interaction in crystal tetracene molecule.25

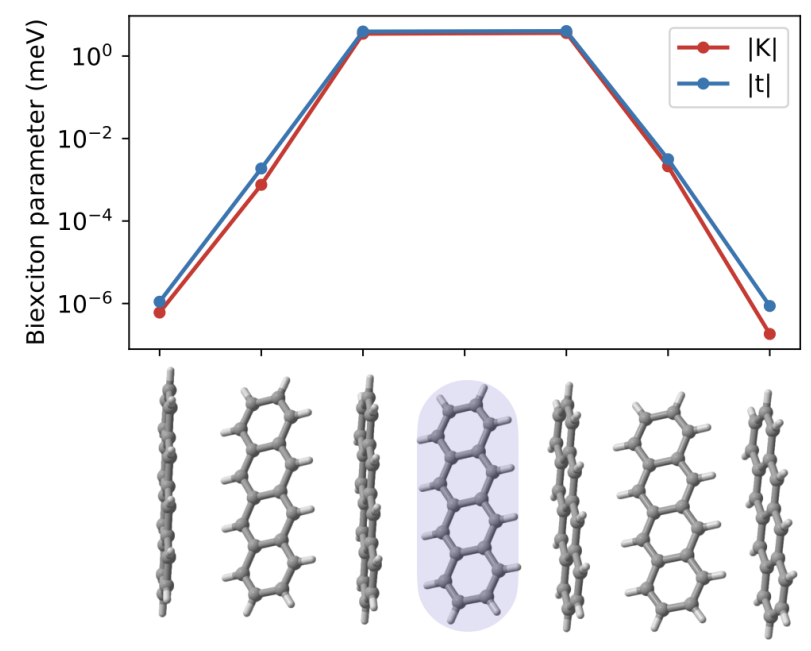

FIG. 5: The exchange and transfer integral for the herringbone structure from a tetracene crystal structure. We present values of $\mathrm{K}$ and $\mathrm{t}$ for the central chromophore (highlighted) with the adjacent chromophores.

\section{B. Biexciton energy manifold for $1 \mathrm{D}$ system}

In this section, we analyse the distance dependence of the exchange and transfer parameters for a herringbone structure of a tetracene lattice. In Figure 5, we present the exchange and hopping parameters that couple the central chromophore with the other chromophores. It can be seen that the exchange and transfer integrals decay exponentially as the distance between the chromophores increases. For the next nearest neighbour dimer, the exchange interaction is on the scale of $\mu \mathrm{eV}$. Because of this, we do not include the next nearest neighbour interactions in our current 1D Hamiltonian model. In the future, however, when considering spin mixing interactions, these need to be included since spin dipolar interactions are also in the $\mu \mathrm{eV}$ range. ${ }^{22141}$ Each of these couplings are computed using a single spin flip CAS calculation.

We generate the biexciton energy manifold for a model 1D system with three chromophores. With three chromophores, there are total $3 \times{ }^{3} C_{2}=3 \times 3$ biexciton states. We analyze the effect of varying $K$ and $t$ on the biexciton spectrum.

We present the biexciton energy values for both antiferromagnetic and ferromagnetic exchange interactions $(K)$ in Figure 6. The sign of $t$ does not change the biexciton spectrum. We fix the $K$ parameter and vary $t$ on the right side of the grey line and on the left we fix $t$ and vary $K$ for both antiferromagnetic (Figure 6a) and ferromagnetic (Figure 6p) $K$. For the antiferromagentic case with very low $t$, it can be seen that the ${ }^{1}(\mathrm{TT})$ state is much lower in energy than the ${ }^{3}(\mathrm{TT})$ and ${ }^{5}(\mathrm{TT})$ states since $K$ value dominates here.

As we increase the $t$ parameter, we can see that one of each $^{1}(\mathrm{TT}),{ }^{3}(\mathrm{TT})$ and ${ }^{5}$ (TT) states becomes more stable until $K=t$. When $K=0$, all three spin states are degenerate and would facilitate spin mixing. As we increase the $K$ parameter, the ${ }^{1}(\mathrm{TT})$ state becomes lowest energy for the antiferromagnetic case and the ${ }^{5}$ (TT) becomes lowest energy for the ferromagnetic case. The spectrum is flipped vertically for the ferromagnetic $K$ values. Having the ${ }^{5}(\mathrm{TT})$ lower in energy would improve the final triplet yield since the ${ }^{5}(\mathrm{TT})$ state does not have other decay pathways. Hence for ideal singlet fission dissociation, low/negative $K$ values would be favourable such that ${ }^{1}(\mathrm{TT})$ and ${ }^{5}(\mathrm{TT})$ are very close in energy.

In the future, we will include the spin dipole interactions into the model to investigate the effect of spin mixing. A kinetic model with the diabatic states for (TT) and $(\mathrm{T} \ldots \mathrm{T})$ including both biexciton transfer and biexciton exchange can shed more light into the biexciton dynamics and will be investigated in future work. Geometric relaxation is also needed for a complete description of the mechanism 86

\section{Biexciton coupling for tetracene dimer}

The decoherence of the ${ }^{1}$ (TT) largely depends on the $K$ and $t$ parameters for the model presented. While large $K$ leads to longer coherence time, large $t$ helps in the formation of the (T...T) state. In this section, we investigate how these parameters vary as the molecular pack- 
(a)

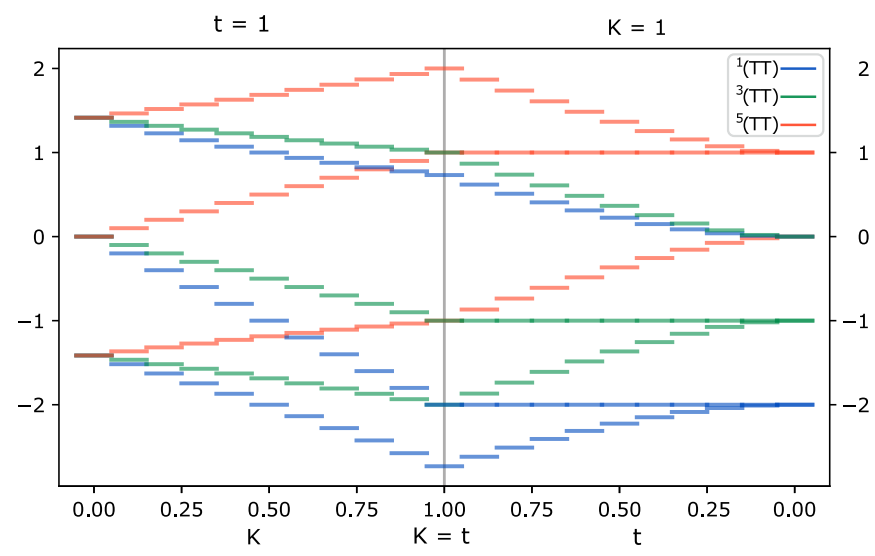

(b)

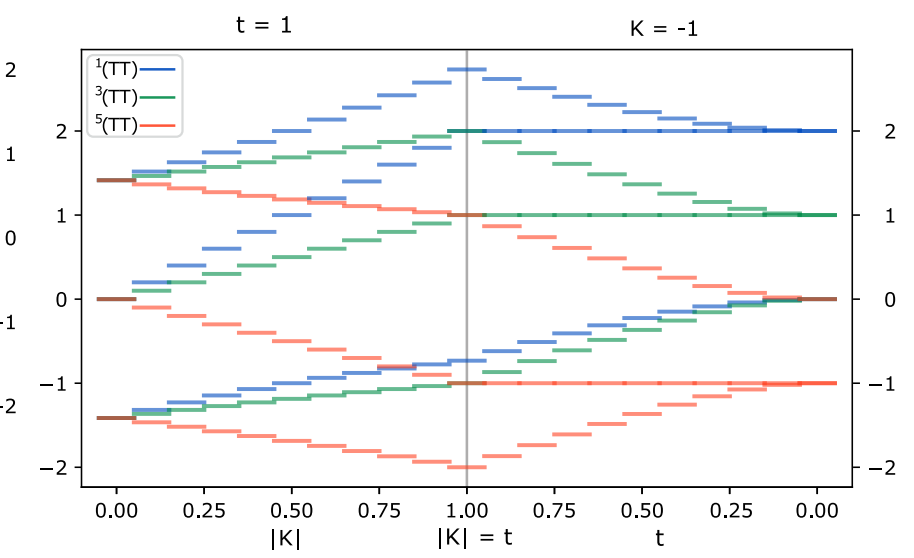

FIG. 6: Comparison of the biexciton energy manifold for varying values of $K$ and $t$ for a) antiferromagnetic (positive $K$ ) and b) ferromagnetic (negative $K$ ) exchange interactions. We fix $t=1 \mathrm{meV}$ for the left of the vertical gray line and vary $K$ uniformly from 0 to 1 . On the right side of gray line, we vary the $t$ parameter from 1 to 0 .

ing is changed. The chromophore dimer units are generated using optimized geometries of tetracene molecules at B3LYP/6-31G(d). We present $t$ and $K$ parameters as a function of chromophore orientation.

a. Center of mass-coordinate In this first panel, Figure 7 (a), the location of one chromophore is fixed and the other chromophore is translated along the $\mathrm{z}$ axis. The $K$ and $t$ parameters decay exponentially as the distance between the chromophores $(d)$ increases with exponential factors of $e^{-2.76} d$ and $e^{-2.77} d$, respectively. Beyond $5 \stackrel{\circ}{A}$ the couplings fall below $1 \mathrm{meV}$. Hence having a large distance between the chromophores can increase spin mixing as the only non-exponentially decaying term remaining are spin dipolar interactions. Since both $t$ and $K$ decay similarly as a function of center of mass separation (the $K / t$ ratio stays fairly constant at around 0.73 ), therefore this is not a useful coordinate for optimizing triplet-triplet separation.

b. $\theta$-coordinate The energetically favorable herringbone structure of simple chromophores stabilize dimers which are rotated with respect to each other. Here we analyze how this rotation angle coordinate between the two chromophores influences the biexciton exchange and transfer integrals. We fix the distance between the centre of mass of the two molecules to be $5.0 \AA$ and rotate one of the chromophores. Since the center of mass is fixed, by rotating we are bringing the two chromophores closer, hence at larger angles the parameters increase in magnitude, although they are still less than $10 \mathrm{meV}$. From Figure 7(b), it can be seen that the exchange interaction between the two chromophores becomes very low at about 64 degrees. This is the geometry were we have realized the smallest $K / t$ ratio, which (based on the considerations discussed above), should provide the fastest ${ }^{1}(\mathrm{TT}) \rightarrow(\mathrm{T} \ldots \mathrm{T})$ separation and decoherence.

Consistent with models based on orbital overlap 87 , the perpendicular geometry drives the triplet hopping term becomes zero, essentially shutting down any formation of $(\mathrm{T} \ldots \mathrm{T})$. The spin lattice in the case of the exact perpendicular geometry is similar to a orthogonal dimer arrangement which is basically a 1D Shastry-Sutherland spin lattice. 88

c. $x$-coordinate Next, we study the effect on biexciton exchange and transfer integrals when one chromophore is moved in the $\mathrm{x}$ direction (Figure 7(c)). We fix the distance between the planes of the two chromophores to be $3.75 \AA$. We can see that there is an interesting variation of $K$ and $t$ in this case and the periodicity is almost similar. While the sign of $t$ oscillates along this coordinate, this does not affect the hopping rate since it depends on $t^{2} \stackrel{32 / 40}{ }$ In terms of minimizing $K / t$, one can see a favorable region occurring at around $d=2.75 \AA$ where $t$ reaches a local maximum. The exchange interaction between the two triplet states is ferromagnetic for the x shift from $5 \AA$ to $5.6 \AA$ and $7.6 \AA$ to $8.2 \AA$. For these geometries, the ${ }^{5}$ (TT) state is lower in energy compared to the ${ }^{1}$ (TT) state.

d. $y$-coordinate We also study the parameters as we move the chromophore in the y direction (Figure $7(\mathrm{~d})$ ). We fix the distance between the two chromophores to be $3.5 \AA$ (similar results were obtained at $3.75 \AA$ ). As seen in the previous case, the values of the $t$ and $K$ parameters have similar periodicity. In terms of $K / t$ ratio, this coordinate minimizes this ratio only at small values of $d$, where the two chromophores are directly on top of one another. Hence we do not expect fast dissociation in any of the geometries along this coordinate.

\section{CONCLUSION}

In this work we present a simple biexciton model for the correlated triplet pair state intermediates in singlet fission materials. The biexciton energy manifold is 
(a)

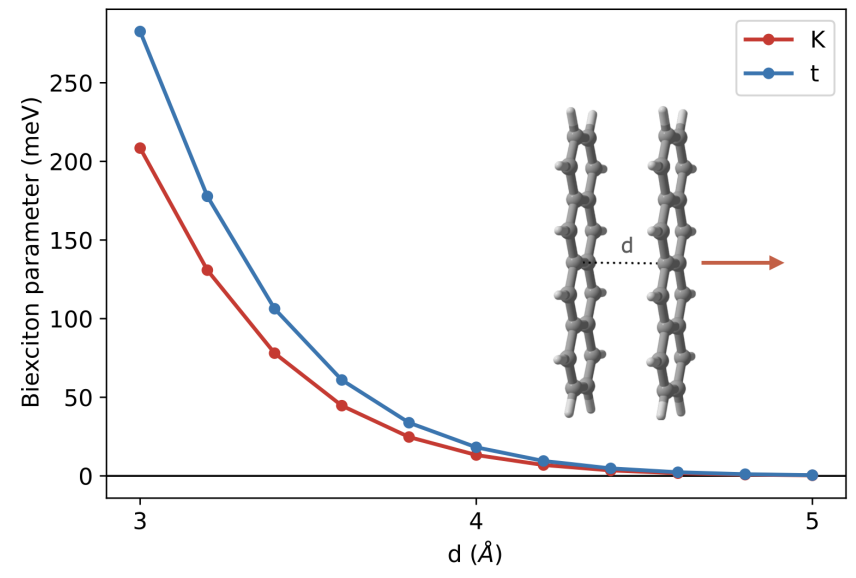

(c)

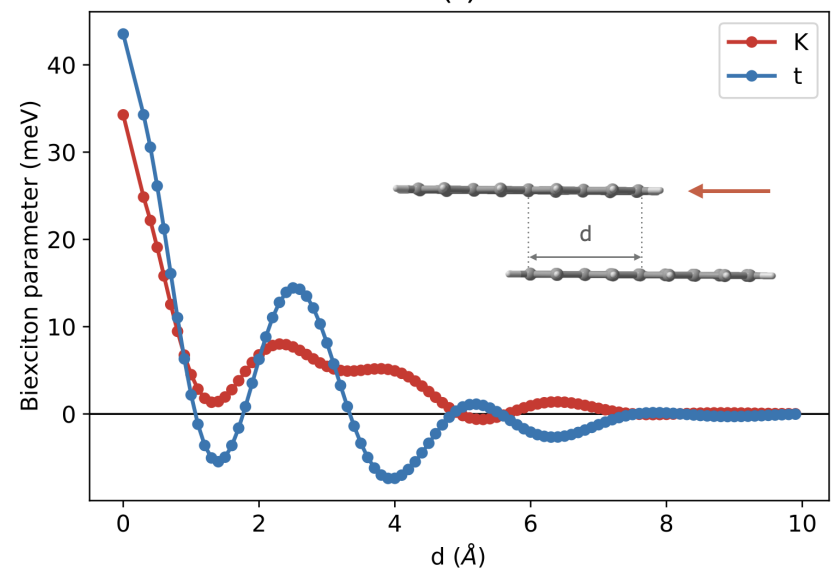

(b)

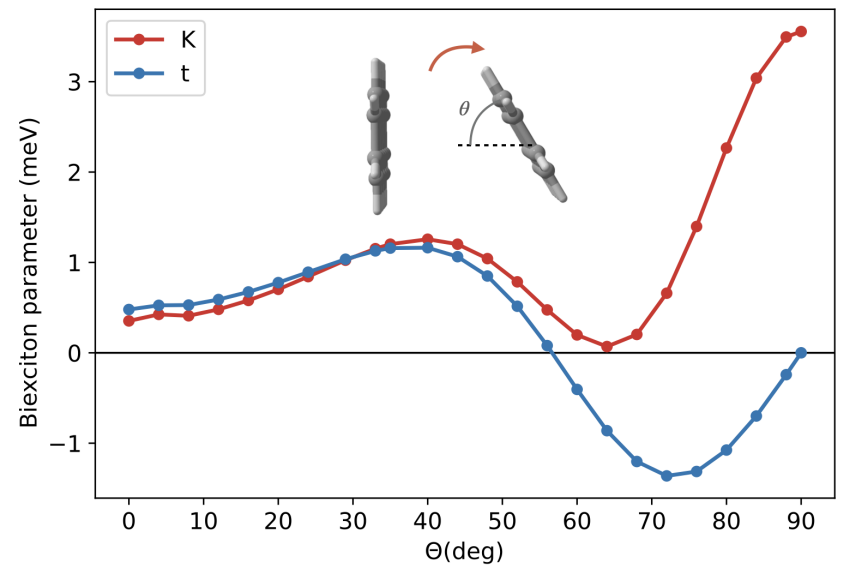

(d)

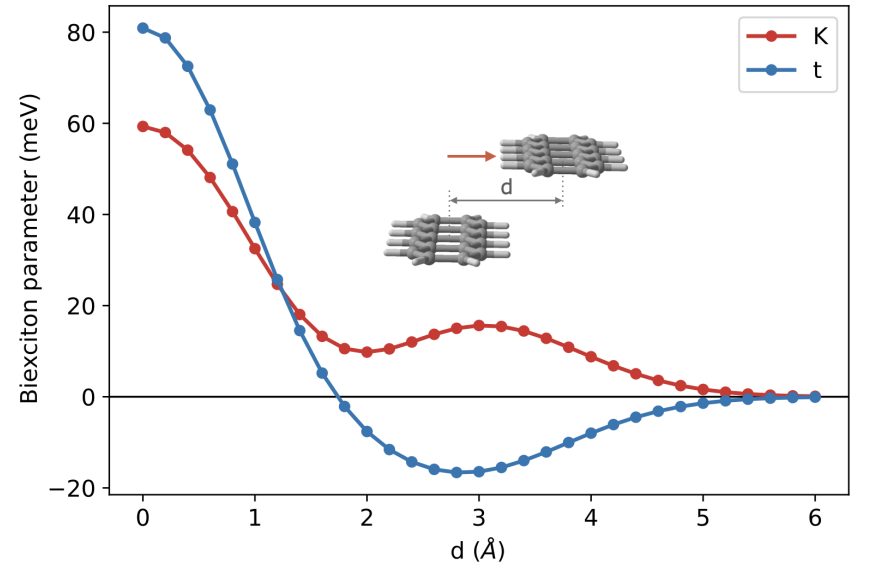

FIG. 7: The exchange and transfer coupling for the tetracene dimer.

mainly decided by two key parameters, the exciton transfer term $(t)$ and the triplet-triplet exchange interaction $(K)$. We demonstrate that these parameters can be derived from a simple ab initio calculation by using a single spin flip calculation from a high spin reference using the 1SF-Bloch approach as presented in this work. $K$ and $t$ are competing interactions, for the antiferromagnetic exchange, $K>>t$ can lead to bound biexcitons whereas $t>>K$ leads to small energy gap between ${ }^{1}(\mathrm{TT})$ and ${ }^{5}(\mathrm{TT})$, and favourable formation of ${ }^{1}(\mathrm{~T} \ldots \mathrm{T})$ states. For a ferromagnetic exchange, the quintet biexciton states is more stable than the singlet state even for larger chromophore assemblies and hence can lead to higher triplet yield $\frac{17 / 26}{2}$

These different ratios of $K$ and $t$ depend on chromophore interactions, hence we investigate the effect of molecular packing. For most of the geometries, the $K$ value is antiferromagnetic. We find ferromagnetic interaction for a small region when the two chromophores are shifted along the $\mathrm{x}$ axis around $\sim 5.3 \AA$ and $\sim 7.9 \AA$. Very few chromophore arrangements lead to conditions where $t$ is significantly larger than $K$. We find large exciton transfer compared to exchange along the $\theta$ rotation coordinate around 60 degrees. For these molecular packings, the biexciton dissociation should be favoured either by the fast formation of ${ }^{1}(\mathrm{~T} \ldots \mathrm{T})$ state or the ${ }^{5}(\mathrm{TT})$ state.

In the future, we will be developing a Marcus theory based model which includes the entanglement of the triplet pair states ${ }^{32}$ The $1 \mathrm{SF}-$ Bloch model can be further improved by introducing hole and particle excita-

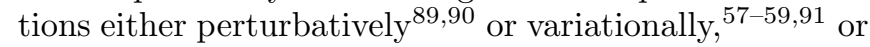
using an effective Hamiltonian derived using equation of motion coupled cluster (EOM-CC) ${ }^{[22}$ An intramolecular vibrational model has also been proposed to be important for the formation of the correlated triplet pair states ${ }^{93}$ Vibrational effects can be introduced for the model either by extracting the exchange parameters at different geometries of the vibrational mode or by introducing the vibrational coupling explicitly using phonon Hamiltonian. The 1SF-Bloch model can also be used for obtaining the coupling in triplet-triplet energy transfer reactions for artificial photosynthesis. $\frac{9495}{}$ 


\section{SUPPORTING INFORMATION}

We provide data for the effect of QDPT, FSD scheme for triplet energy transfer, effect of dihedral, effect of system size in the supporting information.

\section{ACKNOWLEDGEMENTS}

This research was supported by the National Science Foundation (Award No. 1752612). The authors acknowledge Advanced Research Computing at Virginia Tech for providing computational resources and technical support that have contributed to the results reported within this paper. URL: https://arc.vt.edu/

\section{References}

[1] Smith, M. B.; Michl, J. Singlet fission. Chem. Rev. 2010, 110, 6891-6936.

[2] Smith, M. B.; Michl, J. Recent Advances in Singlet Fission. Annu. Rev. Phys. Chem. 2013, 64, 361-386, PMID: 23298243.

[3] Casanova, D. Theoretical Modeling of Singlet Fission. Chem. Rev. 2018, 118, 7164-7207, PMID: 29648797.

[4] Ullrich, T.; Munz, D.; Guldi, D. M. Unconventional singlet fission materials. Chem. Soc. Rev. 2021, 50, 34853518.

[5] Hanna, M. C.; Nozik, A. J. Solar conversion efficiency of photovoltaic and photoelectrolysis cells with carrier multiplication absorbers. J. Appl. Phys. 2006, 100, 074510.

[6] Miyata, K.; Conrad-Burton, F. S.; Geyer, F. L.; Zhu, X.Y. Triplet Pair States in Singlet Fission. Chem. Rev. 2019, 119, 4261-4292.

[7] Sanders, S. N.; Pun, A. B.; Parenti, K. R.; Kumarasamy, E.; Yablon, L. M.; Sfeir, M. Y.; Campos, L. M. Understanding the Bound Triplet-Pair State in Singlet Fission. Chem 2019, 5, 1988-2005.

[8] Musser, A. J.; Clark, J. Triplet-Pair States in Organic Semiconductors. Annu. Rev. Phys. Chem. 2019, 70, 323351, PMID: 31174458.

[9] Kim, H.; Zimmerman, P. M. Coupled double triplet state in singlet fission. Phys. Chem. Chem. Phys. 2018, 20, 30083-30094.

[10] Zimmerman, P. M.; Zhang, Z.; Musgrave, C. B. Singlet fission in pentacene through multi-exciton quantum states. Nat. Chem. 2010, 2, 648-652.

[11] Sanders, S. N.; Kumarasamy, E.; Pun, A. B.; Trinh, M. T.; Choi, B.; Xia, J.; Taffet, E. J.; Low, J. Z.; Miller, J. R.; Roy, X.; Zhu, X.-Y.; Steigerwald, M. L.; Sfeir, M. Y.; Campos, L. M. Quantitative Intramolecular Singlet Fission in Bipentacenes. J. Am. Chem. Soc. 2015, 137, 8965-8972, PMID: 26102432.

[12] Tayebjee, M. J. Y.; Sanders, S. N.; Kumarasamy, E.; Campos, L. M.; Sfeir, M. Y.; McCamey, D. R. Quintet multiexciton dynamics in singlet fission. Nat. Phys. 2017, 13, 182-188.

[13] Folie, B. D.; Haber, J. B.; Refaely-Abramson, S.; Neaton, J. B.; Ginsberg, N. S. Long-Lived Corre- lated Triplet Pairs in a $\pi$-Stacked Crystalline Pentacene Derivative. J. Am. Chem. Soc. 2018, 140, 2326-2335.

[14] Kundu, A.; Dasgupta, J. Photogeneration of Long-Lived Triplet States through Singlet Fission in Lycopene HAggregates. J. Phys. Chem. Lett 2021, 12, 1468-1474, PMID: 33528257.

[15] Zhou, Z.; Ma, L.; Guo, D.; Zhao, X.; Wang, C.; Lin, D.; Zhang, F.; Zhang, J.; Nie, Z. Ultrafast Dynamics of LongLived Bound Triplet Pair Generated by Singlet Fission in 6,13-Bis(triisopropylsilylethynyl) Pentacene. J. Phys. Chem. C. 2020, 124, 14503-14509.

[16] Basel, B. S. et al. Unified model for singlet fission within a non-conjugated covalent pentacene dimer. Nat. Commun. 2017, 8, 1-8.

[17] Sakai, H.; Inaya, R.; Nagashima, H.; Nakamura, S.; Kobori, Y.; Tkachenko, N. V.; Hasobe, T. Multiexciton Dynamics Depending on Intramolecular Orientations in Pentacene Dimers: Recombination and Dissociation of Correlated Triplet Pairs. J. Phys. Chem. Lett 2018, 9, 3354-3360, PMID: 29847939.

[18] Lubert-Perquel, D.; Salvadori, E.; Dyson, M.; Stavrinou, P. N.; Montis, R.; Nagashima, H.; Kobori, Y.; Heutz, S.; Kay, C. W. M. Identifying triplet pathways in dilute pentacene films. Nat. Commun. 2018, 9, 4222.

[19] Nagashima, H.; Kawaoka, S.; Akimoto, S.; Tachikawa, T.; Matsui, Y.; Ikeda, H.; Kobori, Y. Singlet-Fission-Born Quintet State: Sublevel Selections and Trapping by Multiexciton Thermodynamics. J. Phys. Chem. Lett. 2018, 9, 5855-5861.

[20] Weiss, L. R.; Bayliss, S. L.; Kraffert, F.; Thorley, K. J.; Anthony, J. E.; Bittl, R.; Friend, R. H.; Rao, A.; Greenham, N. C.; Behrends, J. Strongly exchange-coupled triplet pairs in an organic semiconductor. Nat. Phys. 2017, 13, 176-181.

[21] Chen, M.; Krzyaniak, M. D.; Nelson, J. N.; Bae, Y. J.; Harvey, S. M.; Schaller, R. D.; Young, R. M.; Wasielewski, M. R. Quintet-triplet mixing determines the fate of the multiexciton state produced by singlet fission in a terrylenediimide dimer at room temperature. Proc. Natl. Acad. Sci. 2019, 116, 8178-8183.

[22] Smyser, K. E.; Eaves, J. D. Singlet fission for quantum information and quantum computing: the parallel JDE model. Sci. Rep. 2020, 10, 1-10.

[23] Bhattacharyya, K.; Datta, A. Polymorphism Controlled Singlet Fission in TIPS-Anthracene: Role of Stacking Orientation. J. Phys. Chem. C. 2017, 121, 1412-1420.

[24] Collins, M. I.; McCamey, D. R.; Tayebjee, M. J. Y. Fluctuating exchange interactions enable quintet multiexciton formation in singlet fission. J. Chem. Phys. 2019, 151, 164104.

[25] Mayhall, N. J. From Model Hamiltonians to ab Initio Hamiltonians and Back Again: Using Single Excitation Quantum Chemistry Methods To Find Multiexciton States in Singlet Fission Materials. J. Chem. Theory Comput 2016, 12, 4263-4273, PMID: 27472260.

[26] Abraham, V.; Mayhall, N. J. Simple Rule To Predict Boundedness of Multiexciton States in Covalently Linked Singlet-Fission Dimers. J. Phys. Chem. Lett. 2017, 8, 5472-5478.

[27] Lin, H.-H.; Kue, K. Y.; Claudio, G. C.; Hsu, C.-P. First Principle Prediction of Intramolecular Singlet Fission and Triplet Triplet Annihilation Rates. J. Chem. Theory Comput 2019, 15, 2246-2253, PMID: 30860838.

[28] Wang, X.; Tom, R.; Liu, X.; Congreve, D. N.; Marom, N. 
An energetics perspective on why there are so few triplet-triplet annihilation emitters. J. Mater. Chem. C 2020, 8, 10816-10824, Publisher: The Royal Society of Chemistry.

[29] Khan, S.; Mazumdar, S. Free Triplets Versus Bound Triplet-Triplet Biexciton in Intramolecular Singlet Fission Materials: Structure-Property Correlations. J. Phys. Chem. C. 2020, 124, 1171-1177.

[30] Scholes, G. D. Correlated Pair States Formed by Singlet Fission and Exciton-Exciton Annihilation. J. Phys. Chem. A. 2015, 119, 12699-12705, PMID: 26595530.

[31] Pensack, R. D.; Ostroumov, E. E.; Tilley, A. J.; Mazza, S.; Grieco, C.; Thorley, K. J.; Asbury, J. B.; Seferos, D. S.; Anthony, J. E.; Scholes, G. D. Observation of Two Triplet-Pair Intermediates in Singlet Exciton Fission. J. Phys. Chem. Lett 2016, 7, 2370-2375, PMID: 27281713.

[32] Lee, T. S.; Lin, Y. L.; Kim, H.; Pensack, R. D.; Rand, B. P.; Scholes, G. D. Triplet Energy Transfer Governs the Dissociation of the Correlated Triplet Pair in Exothermic Singlet Fission. J. Phys. Chem. Lett. 2018, 9, 4087-4095.

[33] Dexter, D. L. A theory of sensitized luminescence in solids. J. Chem. Phys. 1953, 21, 836-850.

[34] Korovina, N. V.; Das, S.; Nett, Z.; Feng, X.; Joy, J.; Haiges, R.; Krylov, A. I.; Bradforth, S. E.; Thompson, M. E. Singlet Fission in a Covalently Linked Cofacial Alkynyltetracene Dimer. J. Am. Chem. Soc. 2016, 138, 617-627, PMID: 26693957.

[35] Srimath Kandada, A. R.; Petrozza, A.; Lanzani, G. Ultrafast dissociation of triplets in pentacene induced by an electric field. Phys. Rev. B 2014, 90, 075310.

[36] Skourtis, S. S.; Liu, C.; Antoniou, P.; Virshup, A. M.; Beratan, D. N. Dexter energy transfer pathways. Proc. Natl. Acad. Sci. 2016, 113, 8115-8120.

[37] You, Z.-Q.; Hsu, C.-P. The fragment spin difference scheme for triplet-triplet energy transfer coupling. $J$. Chem. Phys. 2010, 133, 074105.

[38] Devi, L. S.; Al-Suti, M. K.; Dosche, C.; Khan, M. S.; Friend, R. H.; Köhler, A. Triplet energy transfer in conjugated polymers. I. Experimental investigation of a weakly disordered compound. Phys. Rev. B 2008, 78, 045210.

[39] Scholes, G. D. Long-Range Resonance Energy Transfer in Molecular Systems. Annu. Rev. Phys. Chem. 2003, 54, 57-87, PMID: 12471171.

[40] You, Z.-Q.; Hsu, C.-P.; Fleming, G. R. Triplet-triplet energy-transfer coupling: Theory and calculation. $J$. Chem. Phys. 2006, 124, 044506.

[41] Wang, Z.; Liu, H.; Xie, X.; Zhang, C.; Wang, R.; Chen, L.; Xu, Y.; Ma, H.; Fang, W.; Yao, Y.; Sang, H.; Wang, X.; Li, X.; Xiao, M. Free-triplet generation with improved efficiency in tetracene oligomers through spatially separated triplet pair states. Nat. Chem. 2021, 13, 559-567.

[42] Parker, S. M.; Seideman, T.; Ratner, M. A.; Shiozaki, T. Model Hamiltonian Analysis of Singlet Fission from First Principles. J. Phys. Chem. C. 2014, 118, 12700-12705.

[43] Li, X.; Parrish, R. M.; Martínez, T. J. An ab initio exciton model for singlet fission. J. Chem. Phys. 2020, 153, 184116.

[44] Ambrosio, F.; Troisi, A. Singlet fission in linear chains of molecules. J. Chem. Phys. 2014, 141, 204703.

[45] Yang, C.-H.; Hsu, C.-P. First-Principle Characterization for Singlet Fission Couplings. J. Phys. Chem. Lett 2015,
$6,1925-1929$.

[46] Morrison, A. F.; Herbert, J. M. Evidence for Singlet Fission Driven by Vibronic Coherence in Crystalline Tetracene. J. Phys. Chem. Lett 2017, 8, 1442-1448, PMID: 28277682 .

[47] Tempelaar, R.; Reichman, D. R. Vibronic exciton theory of singlet fission. I. Linear absorption and the anatomy of the correlated triplet pair state. J. Chem. Phys. 2017, $146,174703$.

[48] Zeng, T.; Hoffmann, R.; Ananth, N. The Low-Lying Electronic States of Pentacene and Their Roles in Singlet Fission. J. Am. Chem. Soc. 2014, 136, 5755-5764, PMID: 24697685.

[49] Margulies, E. A.; Logsdon, J. L.; Miller, C. E.; Ma, L.; Simonoff, E.; Young, R. M.; Schatz, G. C.; Wasielewski, M. R. Direct Observation of a ChargeTransfer State Preceding High-Yield Singlet Fission in Terrylenediimide Thin Films. J. Am. Chem. Soc. 2017, 139, 663-671, PMID: 27977196.

[50] Berkelbach, T. C.; Hybertsen, M. S.; Reichman, D. R. Microscopic theory of singlet exciton fission. II. Application to pentacene dimers and the role of superexchange. J. Chem. Phys. 2013, 138, 114103.

[51] Berkelbach, T. C. Adv. Chem. Phys; John Wiley \& Sons, Ltd, 2017; Chapter 1, pp 1-38.

[52] Stern, H. L.; Musser, A. J.; Gelinas, S.; Parkinson, P.; Herz, L. M.; Bruzek, M. J.; Anthony, J.; Friend, R. H.; Walker, B. J. Identification of a triplet pair intermediate in singlet exciton fission in solution. Proc. Natl. Acad. Sci. 2015, 112, 7656-7661.

[53] Dreuw, A.; Head-Gordon, M. Single-Reference ab Initio Methods for the Calculation of Excited States of Large Molecules. Chem. Rev. 2005, 105, 4009-4037, PMID: 16277369 .

[54] Roos, B. O.; Taylor, P. R.; Sigbahn, P. E. A complete active space SCF method (CASSCF) using a density matrix formulated super-CI approach. Chem. Phys. 1980, 48, 157-173.

[55] Hirao, K. Multireference Møller-Plesset perturbation treatment of potential energy curve of N2. Int. J. Quantum Chem. 1992, 44, 517-526.

[56] Casanova, D.; Krylov, A. I. Spin-flip methods in quantum chemistry. Phys. Chem. Chem. Phys. 2020, 22, 43264342.

[57] Casanova, D.; Head-Gordon, M. Restricted active space spin-flip configuration interaction approach: theory, implementation and examples. Phys. Chem. Chem. Phys. 2009, 11, 9779-9790.

[58] Zimmerman, P. M.; Bell, F.; Goldey, M.; Bell, A. T.; Head-Gordon, M. Restricted active space spin-flip configuration interaction: Theory and examples for multiple spin flips with odd numbers of electrons. J. Chem. Phys. 2012, 137, 164110.

[59] Bell, F.; Zimmerman, P. M.; Casanova, D.; Goldey, M.; Head-Gordon, M. Restricted active space spin-flip (RASSF) with arbitrary number of spin-flips. Phys. Chem. Chem. Phys. 2013, 15, 358-366.

[60] Zimmerman, P. M.; Bell, F.; Casanova, D.; HeadGordon, M. Mechanism for Singlet Fission in Pentacene and Tetracene: From Single Exciton to Two Triplets. J. Am. Chem. Soc. 2011, 133, 19944-19952, PMID: 22084927.

[61] Casanova, D. Electronic Structure Study of Singlet Fission in Tetracene Derivatives. J. Chem. Theory Comput 
2014, 10, 324-334, PMID: 26579913.

[62] Feng, X.; Luzanov, A. V.; Krylov, A. I. Fission of Entangled Spins: An Electronic Structure Perspective. J. Phys. Chem. Lett 2013, 4, 3845-3852.

[63] Kolomeisky, A. B.; Feng, X.; Krylov, A. I. A Simple Kinetic Model for Singlet Fission: A Role of Electronic and Entropic Contributions to Macroscopic Rates. J. Phys. Chem. C. 2014, 118, 5188-5195.

[64] Jiang, H.; Zimmerman, P. M. Charge transfer via spin flip configuration interaction: Benchmarks and application to singlet fission. J. Chem. Phys. 2020, 153, 064109.

[65] Mayhall, N. J.; Head-Gordon, M. Computational Quantum Chemistry for Multiple-Site Heisenberg Spin Couplings Made Simple: Still Only One Spin-Flip Required. J. Phys. Chem. Lett 2015, 6, 1982-1988.

[66] Benk, H.; Sixl, H. Theory of two coupled triplet states. Molecular Physics 1981, 42, 779-801.

[67] Bloch, C. Sur La Théorie Des Perturbations Des États Liés. Nuclear Physics 1958, 6, 329-347.

[68] Des Cloizeaux, J. Extension D'une Formule De Lagrange À Des Problèmes. Nuclear Physics 1960, 20, 321-346.

[69] Liu, X.; Subotnik, J. E. The Variationally OrbitalAdapted Configuration Interaction Singles (VOA-CIS) Approach to Electronically Excited States. J. Chem. Theory Comput 2014, 10, 1004-1020, PMID: 26580179.

[70] Taffet, E. J.; Beljonne, D.; Scholes, G. D. Overlap-Driven Splitting of Triplet Pairs in Singlet Fission. J. Am. Chem. Soc. 2020, 142, 20040-20047, PMID: 33190497.

[71] Wakasa, M.; Kaise, M.; Yago, T.; Katoh, R.; Wakikawa, Y.; Ikoma, T. What Can Be Learned from Magnetic Field Effects on Singlet Fission: Role of Exchange Interaction in Excited Triplet Pairs. J. Phys. Chem. C 2015, 119, 25840-25844.

[72] Bayliss, S. L.; Chepelianskii, A. D.; Sepe, A.; Walker, B. J.; Ehrler, B.; Bruzek, M. J.; Anthony, J. E.; Greenham, N. C. Geminate and Nongeminate Recombination of Triplet Excitons Formed by Singlet Fission. Phys. Rev. Lett. 2014, 112, 238701.

[73] Wan, Y.; Guo, Z.; Zhu, T.; Yan, S.; Johnson, J.; Huang, L. Cooperative singlet and triplet exciton transport in tetracene crystals visualized by ultrafast microscopy. Nat. Chem. 2015, 7, 785-792.

[74] Srimath Kandada, A. R.; Petrozza, A.; Lanzani, G. Ultrafast dissociation of triplets in pentacene induced by an electric field. Phys. Rev. B 2014, 90, 075310.

[75] Trinh, M. T.; Pinkard, A.; Pun, A. B.; Sanders, S. N.; Kumarasamy, E.; Sfeir, M. Y.; Campos, L. M.; Roy, X.; Zhu, X.-Y. Distinct properties of the triplet pair state from singlet fission. Sci. Adv. 2017, 3, e1700241.

[76] Korovina, N. V.; Pompetti, N. F.; Johnson, J. C. Lessons from intramolecular singlet fission with covalently bound chromophores. J. Chem. Phys. 2020, 152, 040904.

[77] Korovina, N. V.; Chang, C. H.; Johnson, J. C. Spatial separation of triplet excitons drives endothermic singlet fission. Nat. Chem. 2020, 12, 391-398.

[78] Tao, G.; Tan, Y. Modular Tensor Diagram Approach for the Construction of Spin Eigenfunctions: The Case Study of Exciton Pair States. J. Phys. Chem. A. 2020, 124, 5435-5443, PMID: 32551608.

[79] Grieco, C.; Doucette, G. S.; Munro, J. M.; Kennehan, E. R.; Lee, Y.; Rimshaw, A.; Payne, M. M.; Wonderling, N.; Anthony, J. E.; Dabo, I.; Gomez, E. D.; Asbury, J. B. Triplet Transfer Mediates Triplet
Pair Separation during Singlet Fission in 6,13Bis(triisopropylsilylethynyl)-Pentacene. Advanced Functional Materials 2017, 27, 1703929.

[80] Hartzler, D. A.; Slipchenko, L. V.; Savikhin, S. Triplet-Triplet Coupling in Chromophore Dimers: Theory and Experiment. J. Phys. Chem. A. 2018, 122, 67136723, PMID: 30040412.

[81] Yost, S. R.; Hontz, E.; Yeganeh, S.; Van Voorhis, T. Triplet vs Singlet Energy Transfer in Organic Semiconductors: The Tortoise and the Hare. J. Phys. Chem. C. 2012, 116, 17369-17377.

[82] Fujimoto, K. J. Transition-density-fragment interaction combined with transfer integral approach for excitationenergy transfer via charge-transfer states. J. Chem. Phys. 2012, 137, 034101.

[83] qiang You, Z.; Hsu, C.-P. Theory and calculation for the electronic coupling in excitation energy transfer. Int. J. Quantum Chem. 2014, 114, 102-115.

[84] Pun, A. B.; Asadpoordarvish, A.; Kumarasamy, E.; Tayebjee, M. J.; Niesner, D.; McCamey, D. R.; Sanders, S. N.; Campos, L. M.; Sfeir, M. Y. Ultra-fast intramolecular singlet fission to persistent multiexcitons by molecular design. Nat. Chem. 2019, 11, 821-828.

[85] Della Valle, R. G.; Venuti, E.; Brillante, A.; Girlando, A. Inherent Structures of Crystalline Tetracene. J. Phys. Chem. A. 2006, 110, 10858-10862, PMID: 16970382.

[86] Korovina, N. V.; Joy, J.; Feng, X.; Feltenberger, C.; Krylov, A. I.; Bradforth, S. E.; Thompson, M. E. LinkerDependent Singlet Fission in Tetracene Dimers. J. Am. Chem. Soc. 2018, 140, 10179-10190, PMID: 30016102.

[87] Valeev, E. F.; Coropceanu, V.; da Silva Filho, D. A.; Salman, S.; Brédas, J.-L. Effect of Electronic Polarization on Charge-Transport Parameters in Molecular Organic Semiconductors. J. Am. Chem. Soc. 2006, 128, 98829886, PMID: 16866546.

[88] Sriram Shastry, B.; Sutherland, B. Exact ground state of a quantum mechanical antiferromagnet. Physica $B+C$ 1981, 108, 1069-1070.

[89] Mayhall, N. J.; Goldey, M.; Head-Gordon, M. A Quasidegenerate Second-Order Perturbation Theory Approximation to RAS-nSF for Excited States and Strong Correlations. J. Chem. Theory Comput 2014, 10, 589-599, PMID: 26580035.

[90] Mayhall, N. J.; Head-Gordon, M. Increasing spin-flips and decreasing cost: Perturbative corrections for external singles to the complete active space spin flip model for low-lying excited states and strong correlation. J. Chem. Phys. 2014, 141, 044112.

[91] Abraham, V.; Mayhall, N. J. Selected Configuration Interaction in a Basis of Cluster State Tensor Products. J. Chem. Theory Comput. 2020, 16, 6098-6113.

[92] Pokhilko, P.; Krylov, A. I. Effective Hamiltonians derived from equation-of-motion coupled-cluster wave functions: Theory and application to the Hubbard and Heisenberg Hamiltonians. J. Chem. Phys. 2020, 152, 094108.

[93] Tempelaar, R.; Reichman, D. R. Vibronic exciton theory of singlet fission. III. How vibronic coupling and thermodynamics promote rapid triplet generation in pentacene crystals. J. Chem. Phys. 2018, 148, 244701.

[94] Ho, J.; Kish, E.; Méndez-Hernández, D. D.; WongCarter, K.; Pillai, S.; Kodis, G.; Niklas, J.; Poluektov, O. G.; Gust, D.; Moore, T. A.; Moore, A. L.; Batista, V. S.; Robert, B. Triplet-triplet energy transfer in artificial and natural photosynthetic antennas. Proc. 
Natl. Acad. Sci. 2017, 114, E5513-E5521.

[95] Tejeda-Ferrari, M. E.; Brown, C. L.; Coutinho, G. C. C. C.; Gomes de Sá, G. A.; Palma, J. L.; LlansolaPortoles, M. J.; Kodis, G.; Mujica, V.; Ho, J.; Gust, D.; Moore, T. A.; Moore, A. L. Electronic Structure and Triplet-Triplet Energy Transfer in Artificial Photosynthetic Antennas. Photochemistry and Photobiology 2019, 95, 211-219. 